\title{
Estimation of central systolic blood pressure using an oscillometric blood pressure monitor
}

\author{
Hao-Min Cheng ${ }^{1,2}$, Kang-Ling Wang ${ }^{2,3}$, Ying-Hwa Chen ${ }^{2,3}$, Shing-Jong Lin ${ }^{1,4}$, Lung-Ching Chen ${ }^{2,3}$, \\ Shih-Hsien Sung ${ }^{2,3}$, Philip Yu-An Ding ${ }^{2,3}$, Wen-Chung Yu ${ }^{2,3}$, Jaw-Wen Chen ${ }^{1,4}$ and Chen-Huan Chen ${ }^{1,2,4}$
}

Current noninvasive techniques for assessing central aortic pressure require the recording of an arterial pressure wave using a high-fidelity applanation tonometer. We therefore developed and validated a novel method to estimate the central aortic systolic pressure using an oscillometric blood pressure monitor alone. Invasive high-fidelity right brachial and central aortic pressure waves, and left-brachial pulse volume plethysmography from an oscillometric blood pressure monitor, were obtained at baseline and $3 \mathrm{~min}$ after administration of sublingual nitroglycerin in 100 patients during cardiac catheterization. In the initial 50 patients (Generation Group), Central systolic blood pressure was predicted by a multi-variate prediction model generated from the comprehensive analysis of the invasive brachial pressure wave, including brachial late-systolic shoulder pressure value and parameters related to wave reflection and arterial compliance. Another prediction model was similarly constructed from the noninvasively calibrated pulse volume plethysmography. Both models were validated in the subsequent 50 patients (Validation Group) with results: $r=0.98(P<0.001)$ and mean difference $=0.5 \pm 4.5(95 \%$ confidence interval -8.3 to 9.3$) \mathrm{mm} \mathrm{Hg}$ for the invasive model, and $r=0.93(P<0.001)$ and mean difference $=-0.1 \pm 7.6(95 \%$ confidence interval -15.0 to 14.8$) \mathrm{mm} \mathrm{Hg}$ for the noninvasive model. Thus, our results indicate that central aortic systolic blood pressure could be estimated by analysis of the noninvasive brachial pressure wave alone from an oscillometric blood pressure monitor.

Hypertension Research (2010) 33, 592-599; doi:10.1038/hr.2010.37; published online 26 March 2010

Keywords: central blood pressure; oscillometric signals; pressure wave reflection; pulse volume plethysmography

\section{INTRODUCTION}

Aortic and carotid systolic and pulse pressures, commonly referred to as the central blood pressure, may be different from the peripheral pressure in the radial and brachial arteries. ${ }^{1,2}$ The discrepancy between the central and peripheral blood pressures is mainly caused by the central-to-peripheral amplification of the pressure pulse and may magnify with administration of vasoactive agents. ${ }^{2-4}$ Furthermore, recent evidence from epidemiological studies, ${ }^{5,6}$ clinical observation, ${ }^{7}$ and a large clinical trial ${ }^{8}$ suggest that central blood pressure is more relevant than peripheral blood pressure in predicting target organ damage and cardiovascular outcomes. However, the implementation of central blood pressure in the daily practice of hypertension management may require the availability of its reliable measurements to both medical care providers in the clinics and patients at home.

Central blood pressure can be estimated noninvasively from the radial or carotid pressure waves recorded by applanation tonometry. ${ }^{6,9}$ To obtain central blood pressure, the radial pressure wave calibrated with brachial cuff systolic blood pressure (SBP) and diastolic blood pressure (DBP) can be mathematically transformed into a central aortic pressure wave using a generalized transfer function. ${ }^{9,10}$ Alternatively, identification of the late systolic shoulder (SBP2) of the calibrated radial pressure wave, firstly proposed by Takazawa et al, ${ }^{2}$ can be used directly to approximate central SBP without the need for a generalized transfer function. ${ }^{1-14}$ In either approach, a high-fidelity arterial tonometer is required to obtain faithful recording, in addition to an oscillometric blood pressure monitor. Therefore, we developed and validated a novel method to estimate the central aortic SBP using the oscillometric signals from an oscillometric blood pressure monitor.

\section{METHODS}

Study population

Subjects referred for diagnostic catheterization for coronary anatomy through radial approach were potential candidates of the study. Subjects were not included if they had acute coronary syndrome, peripheral arterial disease, rhythms other than normal sinus rhythm, or more than $3 \mathrm{~mm} \mathrm{Hg}$ pressure difference between left and right arms. The enrollment of the study subjects was prospective but not consecutive, depending on the daily schedules of the cardiac catheterization and the investigators. Mid-upper arm circumferences, height, waistline, body weight, and personal medical history were obtained before the cardiac catheterization. The study protocol adhered to the principles of the Declaration of Helsinki and was approved by the Institutional Review Board at Taipei Veterans General Hospital. Informed consent was obtained

${ }^{1}$ Department of Medical Research and Education, Taipei Veterans General Hospital, Taipei, Taiwan; ${ }^{2}$ Department of Medicine, National Yang-Ming University, Taipei, Taiwan; ${ }^{3}$ Department of Research and Education, Taipei Veterans General Hospital, Taipei, Taiwan and ${ }^{4}$ Cardiovascular Research Center, National Yang-Ming University, Taipei, Taiwan Correspondence: Dr C-H Chen, Department of Medical Research and Education, Taipei Veterans General Hospital, No. 201, Sec. 2, Shih-Pai Road, Taipei, Taiwan.

E-mail: chench@vghtpe.gov.tw

Part of the work was presented at 2009 American College of Cardiology Annual Scientific Meeting.

Received 17 December 2009; revised 29 January 2010; accepted 3 February 2010; published online 26 March 2010 
from all patients. A total of 100 subjects fulfilled the inclusion and exclusion criteria and completed the study. Their characteristics are given in Table 1. The first 50 subjects were designated as the Generation Group (age range 36-82 years) to produce the invasive and non-invasive prediction models. The subsequent 50 subjects were prospectively enrolled to serve as the Validation Group (age range 29-90 years) for assessing the performance of the prediction models. The two Groups differed slightly in the arm circumference, diagnosis of hypertension and coronary artery disease and the cuff DBP values (Table 1)

\section{Study protocol}

With the study subject resting on the catheterization table, cuff blood pressure values over both arms were simultaneously obtained to ensure minimal pressure difference between both arms and the absence of peripheral arterial disease, using a commercially available oscillometric blood pressure monitor (VP-2000, Colin Corporation, Komaki, Japan) with cuff size of $11 \times 20 \mathrm{~cm}$, $13 \times 24.5 \mathrm{~cm}$ or $15.5 \times 34 \mathrm{~cm}$, corresponding to arm circumference of 17 to $23 \mathrm{~cm}, 20$ to $32 \mathrm{~cm}$ and 30 to $38 \mathrm{~cm}$, respectively. After taking two consecutive measurements of cuff blood pressure, the blood pressure monitor performed the pulse volume recording, also known as pulse volume plethysmography (PVP), at user-defined levels of cuff pressure. The validity and reproducibility of the device have been documented. ${ }^{15}$ The recorded cuff pressure signals during PVP have been found to correspond to the intra-arterial pressure contours. ${ }^{16}$ The cuff blood pressure readings were the averages of two consecutive measurements.

No sedatives or premedications were used before or during the catheterization procedure. After local injection of 2-3cc $1 \%$ lidocaine and successful placement of a $6 \mathrm{~F}$ arterial sheath in the right radial artery, $2.5 \mathrm{mg}$ verapamil was administered intra-arterially to prevent vasospasm during the catheterization. Subsequently, a 2F micromanometer-tipped catheter (model SPC-320, Millar Instruments, Houston, TX, USA) within a standard $6 \mathrm{~F}$ Judkins coronary artery catheter was advanced until its tip was positioned in the right brachial artery at

Table 1 Subject characteristics

\begin{tabular}{|c|c|c|c|}
\hline Characteristics & $\begin{array}{l}\text { Generation group } \\
\qquad(n=50)\end{array}$ & $\begin{array}{l}\text { Validation group } \\
\qquad(n=50)\end{array}$ & P-value \\
\hline Male sex, \% & 78 & 70 & NS \\
\hline Age, years & $62.1 \pm 12.6$ & $61.6 \pm 13.9$ & NS \\
\hline Height, cm & $163.9 \pm 8.6$ & $162.3 \pm 7.7$ & NS \\
\hline Left arm circumference, $\mathrm{cm}$ & $27.7 \pm 2.4$ & $28.5 \pm 2.7$ & 0.027 \\
\hline Body mass index, $\mathrm{kg} \mathrm{m}^{-2}$ & $25.8 \pm 3.0$ & $26.6 \pm 3.3$ & NS \\
\hline Left ventricular ejection fraction, $\%$ & $55.4 \pm 8.6$ & $55.2 \pm 7.6$ & NS \\
\hline Smoking, \% & 26 & 22 & NS \\
\hline Hypertension, \% & 54 & 68 & 0.043 \\
\hline Type II diabetes mellitus, \% & 14 & 20 & NS \\
\hline Coronary artery disease, \% & 42 & 56 & 0.048 \\
\hline \multicolumn{4}{|l|}{ Baseline blood pressures, $\mathrm{mm} \mathrm{Hg}$} \\
\hline Brachial SBP & $137.4 \pm 20.0$ & $133.3 \pm 19.9$ & NS \\
\hline Brachial MBP & $98.2 \pm 13.2$ & $95.4 \pm 12.5$ & NS \\
\hline Brachial DBP & $73.4 \pm 10.6$ & $70.8 \pm 10.1$ & NS \\
\hline Brachial PP & $63.9 \pm 17.2$ & $62.5 \pm 17.3$ & NS \\
\hline Aortic SBP & $126.2 \pm 20.8$ & $123.8 \pm 20.5$ & NS \\
\hline Aortic MBP & $95.7 \pm 13.3$ & $93.3 \pm 12.9$ & NS \\
\hline Aortic DBP & $72.4 \pm 11.8$ & $69.7 \pm 10.9$ & NS \\
\hline Aortic PP & $53.8 \pm 18.1$ & $54.1 \pm 17.7$ & NS \\
\hline Cuff SBP & $132.8 \pm 18.2$ & $128.8 \pm 18.0$ & NS \\
\hline Cuff MBP & $100.7 \pm 14.9$ & $97.4 \pm 14.2$ & NS \\
\hline Cuff DBP & $77.0 \pm 12.1$ & $72.9 \pm 10.3$ & 0.011 \\
\hline Cuff PP & $55.9 \pm 10.3$ & $55.9 \pm 11.7$ & NS \\
\hline Baseline heart rate, beats/min & $70.4 \pm 13.0$ & $67.2 \pm 11.1$ & NS \\
\hline
\end{tabular}

Abbreviations: DBP, diastolic blood pressure; MBP, mean blood pressure; NS, non-significant; PP, pulse pressure; SBP, systolic blood pressure. the level of mid-humerus. The micromanometer has a flat frequency response from 0 to $1000 \mathrm{~Hz}$. The frequency range of our catheterization laboratory amplifier is $0-400 \mathrm{~Hz}$ for pressure measurement $(-50$ to $400 \mathrm{~mm} \mathrm{Hg}$ ) with the accuracy of $\pm 1 \mathrm{~mm} \mathrm{Hg}$ or $\pm 3 \%$ exclusive of transducer. Baseline invasive brachial pressure waves were recorded for consecutive 20-30 beats to cover at least two respiratory cycles. Afterwards, the catheter was advanced further to the ascending aorta in less than $10 \mathrm{~s}$. The central aortic pressure waves were then recorded simultaneously with left-arm PVP performed at mean cuff pressure of $60 \mathrm{~mm} \mathrm{Hg}$ for $30 \mathrm{~s}$, followed by automatic measuring of the left brachial cuff blood pressures. Subsequently, a sublingual nitroglycerin (NTG) was administered. Three minutes later, the left brachial cuff blood pressures were measured again, followed by simultaneous recording of the central aortic pressure waves and left-arm PVP. Immediately after the completion of the post-NTG PVP, the micromanometer catheter was pulled back until its tip was at the level of mid-humerus for recording of 20-30 beats of invasive brachial pressure waves, and then was removed to continue the routine diagnostic coronary angiography. The study procedure, from cannulation of right radial artery to removal of the micromanometer catheter, could be completed in less than $10 \mathrm{~min}$. All signals were digitized instantaneously at a sampling frequency of $250 \mathrm{~Hz}$ for off-line analysis. Left ventricular ejection fraction (LV EF) was routinely measured from single-plane left ventriculogram after diagnostic coronary angiography.

\section{Data analysis}

The digitized signals were analyzed using custom-designed software on a commercial software package (Matlab, version 4.2, The MathWorks, Natick, MA, USA). All processed individual signals were subject to fully automatic batch analysis to avoid inter- and intra-observer variations. Consecutive 20-30 beats of the sequentially derived brachial and aortic pressure waves were signal averaged to one beat, respectively. Aortic SBP and DBP were the peak value and value at end-diastole of the averaged pressure wave, respectively, and the difference was aortic pulse pressure (PP). Aortic mean blood pressure (MBP) was determined from the total area under the averaged pressure curve. Heart rate was calculated from the cardiac cycle length, which was equivalent to the length of the pressure curve. Brachial SBP, DBP, PP, and MBP were similarly obtained from the averaged brachial pressure wave.

Because arterial wave reflection and arterial compliance are the major determinants of the central-to-peripheral amplification of the pressure pulse, ${ }^{3}$ the brachial pressure wave was further comprehensively analyzed to identify parameters, which are associated with arterial wave reflection and arterial compliance. SBP2 resulting from the arterial wave reflection was determined from the zero-crossing timings of the fourth derivative of the pressure wave. ${ }^{2}$ Based on a two-element Windkessel model assuming a linear pressure volume relation, total arterial compliance (C) can be estimated from a central aortic pressure wave using the equation:

$$
\mathrm{C}=\mathrm{SV} /[\mathrm{k}(\mathrm{Pes}-\mathrm{Pd})]
$$

where $\mathrm{SV}=$ stroke volume, $\mathrm{Pes}=$ pressure at onset of diastole, $\mathrm{Pd}=$ pressure at end-diastole, and $k$ was the sum of areas under the pressure tracing in diastole (Ad) and in systole (As) divided by Ad. ${ }^{17}$ Except for SV, the remaining parameters, including Pes, $\mathrm{Pd}$, Ad and As, are obtained from a central aortic pressure wave. Pes, Pd, Ad and As were therefore considered as correlates of C. We have found that Pes, Pd, Ad, and As obtained from a brachial pressure wave can also be used to calculate $\mathrm{C}$ with good correlation $(r>0.9, P<0.01$, unpublished data). Therefore, brachial Pes, Pd, Ad and As were also considered as correlates of $\mathrm{C}$ (Figure 1).

Brachial DBP was considered as the pressure at end-diastole. Brachial endsystolic pressure (ESP) (Figure 1a) was determined from a proprietary algorithm involving the first derivative of the pressure wave. The areas under the brachial pressure curve in diastole (Ad) and systole (As) (Figure la) were measured separately. All parameters, including SBP2, brachial DBP, ESP, Ad, As and heart rate derived from the analysis of the invasive brachial pressure wave were potential components to be used to construct a multivariate prediction model to estimate the invasively measured central aortic SBP.

Consecutive 20-30 beats of left brachial PVP obtained simultaneously with the aortic pressure waves were signal averaged. To develop the noninvasive 

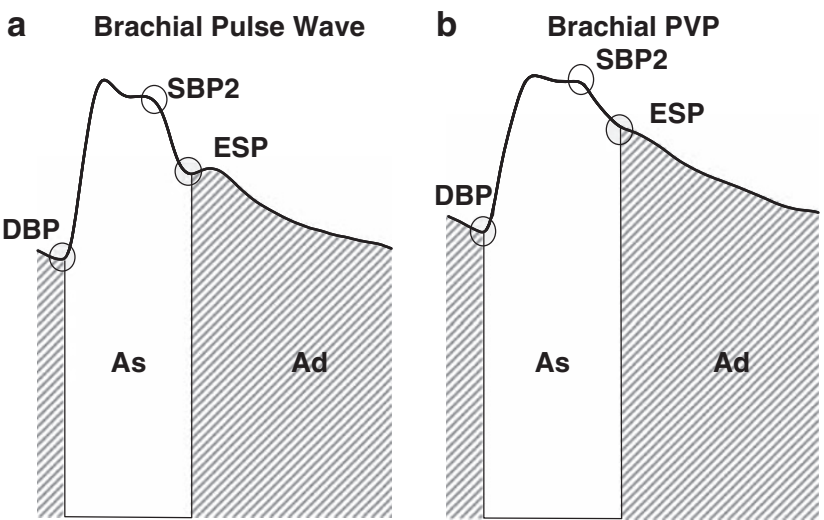

Figure 1 Parameters identified from the brachial pulse (a) and the pulse volume plethysmography (PVP) (b) waveforms for the prediction of aortic systolic blood pressure. Ad, the area under curve during diastole; As, the area under curve during systole; DBP, pressure value at end-diastole; ESP, end-systolic pressure, pressure value at onset of diastole; SBP, systolic blood pressure; SBP2, pressure value of the late systolic shoulder produced by wave reflections.

prediction model, the averaged PVP was calibrated by matching the oscillometric cuff SBP and DBP. The calibrated averaged PVP wave was analyzed using techniques (Figure 1b) similar to those for the analysis of the brachial pressure wave.

To validate the accuracy of the automated identification of SBP2 and ESP on the PVP wave, we timed the intervals from wave foot to peak of the reflection wave and from wave foot to end-systole on the simultaneously recorded aortic pressure and then applied the measured intervals directly to the PVP wave to identify the reference SBP2 and end-systolic pressure. ${ }^{12}$

\section{Statistical analyses}

Correlation coefficients between the parameters from the invasive and noninvasive brachial pulse wave analysis and the invasive aortic SBP were calculated. The statistical significance of the difference between two correlation coefficients was examined by calculating the $z$-statistic. ${ }^{18}$ Stepwise multiple linear regression analysis was used to select the best parameters from the Generation Group to construct the invasive and noninvasive multivariate prediction models for the estimation of the invasive aortic SBP. Comparisons of paired blood pressure values and their differences were performed using paired Student's $t$-test. Agreements between the measured and predicted aortic SBP values were examined using the Bland-Altman analysis. The effects of age, sex, height, weight, waistline, LV EF, heart rate, arm circumference and use of NTG on the performance of the prediction models were examined by multivariate linear regression analysis. Statistical significance is declared at the twotailed $P<0.05$ level.

\section{RESULTS}

Generation of the invasive prediction model (Generation group, $\boldsymbol{n}=\mathbf{5 0}$ )

All parameters except for heart rate from the analysis of the invasive brachial pressure wave, including SBP, MBP, DBP, SBP2, ESP, As and Ad, correlated significantly with the invasive aortic SBP (Supplementary Table S1). A multivariate prediction model to predict the aortic SBP at steady state and after NTG using significant independent parameters from the invasive brachial pressure wave analysis was constructed as follows:

$$
\begin{aligned}
\text { Predicted aortic SBP }= & 0.690 \times \mathrm{SBP} 2+0.440 \times \mathrm{ESP} \\
& +0.157 \times \mathrm{As}-0.223 \times \mathrm{Ad}-2.298
\end{aligned}
$$

The full model $\mathrm{R}^{2}$ was 0.96 and the partial $\mathrm{R}^{2}$ for SBP2, ESP, As and Ad were $0.92,0.02,0.01$ and 0.01 , respectively. Thus, the relative contributions from SBP2 and the remaining components as a whole of the waveform analysis to the explained variance of the model were $95.8 \%(0.92 / 0.96)$ and $4.2 \%$, respectively. Addition of age, sex, height, weight, arm circumference, LV EF or heart rate to the model did not significantly improve the prediction (data not shown).

\section{Generation of the noninvasive prediction model (Generation Group, $n=50$ )}

All parameters except for heart rate from the analysis of the PVP waveforms calibrated by the cuff SBP and DBP, including SBP, MBP, DBP, SBP2, ESP, As and Ad, correlated significantly with the invasive aortic SBP (Supplementary Table S1). A second multivariate prediction model was constructed as follows:

$$
\begin{aligned}
\text { Predicted aortic SBP }= & 0.465 \times \mathrm{SBP} 2+0.465 \times \mathrm{ESP} \\
& +0.490 \times \mathrm{As}-0.069 \times \mathrm{Ad}-0.753
\end{aligned}
$$

The full model $R^{2}$ was 0.85 and the partial $R^{2}$ for SBP2, ESP, As and Ad were $0.78,0.05,0.01$ and 0.01 , respectively. The relative contributions from SBP2 and other components of the waveform analysis to the explained variance of the model were $91.8 \%(0.78 / 0.85)$ and $8.2 \%$, respectively.

Validation of the prediction models (validation group, $n=50$ ) In another 50 study subjects, brachial, aortic and oscillometric SBP declined by $10.1 \pm 12.7 \mathrm{~mm} \mathrm{Hg}, \quad 13.4 \pm 15.1 \mathrm{~mm} \mathrm{Hg}$ and $10.0 \pm 12.5 \mathrm{~mm} \mathrm{Hg}$, respectively, after NTG. The invasive brachial SBP, MBP and PP were significantly higher than the corresponding aortic SBP, MBP and PP, and the brachial-aortic pressure differences magnified substantially for SBP and PP after NTG (Table 2). In contrast, the invasive brachial SBP2 approximated aortic SBP at baseline and after NTG with a relatively constant small overestimation (Table 2).

Cuff blood pressures significantly underestimated brachial SBP and PP at baseline and after NTG (Table 2). Overall, a bias of $4.5 \mathrm{~mm} \mathrm{Hg}$ and the limits of agreement of -21.2 to $12.2 \mathrm{~mm} \mathrm{Hg}$ were derived for the cuff SBP $v s$. the brachial SBP when data at baseline and after NTG were combined. Similar to brachial SBP, cuff SBP significantly overestimated aortic SBP and the overestimation magnified after NTG. The difference between cuff PP and aortic PP also magnified after NTG (Table 2). Although the mean differences between PVP SBP2 and aortic SBP were small at baseline and after NTG, the s.d. for the difference were large (Table 2).

The bias and confidence limits of the estimation for aortic SBP by brachial and cuff SBP and SBP2 and the prediction models are summarized in Table 3. Although the invasive brachial SBP significantly correlated with the invasive aortic SBP, a large bias and wide limits of agreement were observed (Table 3, Figure 2a). The bias and the agreement limits substantially reduced with brachial SBP2 (Table 3, Figure 2b). With the invasive model, the bias and the agreement limits further reduced (Table 3). The mean difference between the predicted and observed aortic SBP was not significantly greater than $0 \mathrm{~mm} \mathrm{Hg}$, and was significantly smaller than that between brachial SBP2 and aortic SBP at baseline and after NTG (all $P<0.001$ ) (Table 3, Figure 2c). The correlation between the measured and predicted aortic SBP by the invasive prediction model (Figure 2c) was significantly better than that by invasive SBP2 alone (Figure 2b) $(P<0.001)$. In addition, the Bland-Altman analysis indicated that there was no systemic bias of estimation with different levels of estimated values. 
Table 2 Comparisons between aortic, brachial, cuff and calibrated pulse volume plethysmography pressure values in the validation group $(n=50)$

\begin{tabular}{|c|c|c|c|c|}
\hline Pressure difference, $\mathrm{mm} \mathrm{Hg}$ & Mean \pm s.d. & $95 \% \mathrm{Cl}$ & Mean \pm s.d. & $95 \% \mathrm{Cl}$ \\
\hline Brachial SBP_aortic SBP & $7.8 \pm 5.3^{* * *}$ & $-2.6 \sim 18.2$ & $11.1 \pm 6.9^{* * *}$ & $-2.4 \sim 24.6$ \\
\hline Brachial MBP_aortic MBP & $2.3 \pm 3.1^{* * *}$ & $-3.8 \sim 8.4$ & $2.0 \pm 4.5^{* *}$ & $-6.8 \sim 10.8$ \\
\hline Brachial DBP_aortic DBP & $1.4 \pm 3.9^{*}$ & $-6.2 \sim 9.0$ & $0.7 \pm 3.7$ & $-6.6 \sim 8.0$ \\
\hline \multicolumn{5}{|l|}{ Cuff pressure-brachial pressure } \\
\hline Cuff SBP—brachial SBP & $-4.5 \pm 7.2^{* * *}$ & $-18.6 \sim 9.6$ & $-4.5 \pm 9.7^{* *}$ & $-23.5 \sim 14.5$ \\
\hline Cuff MBP_brachial MBP & $2.7 \pm 7.7^{\star}$ & $-12.4 \sim 17.8$ & $1.2 \pm 6.9$ & $-12.3 \sim 14.7$ \\
\hline Cuff DBP—brachial DBP & $3.7 \pm 6.1^{* * *}$ & $-8.3 \sim 15.7$ & $0.6 \pm 8.1$ & $-15.3 \sim 16.5$ \\
\hline Cuff PP—brachial PP & $-8.2 \pm 7.8^{* * *}$ & $-23.5 \sim 7.1$ & $-5.0 \pm 13.3^{*}$ & $-31.1 \sim 21.1$ \\
\hline \multicolumn{5}{|l|}{ Cuff pressure - aortic pressure } \\
\hline \multicolumn{5}{|l|}{ PVP pressure-aortic pressure } \\
\hline PVP SBP2-aortic SBPa & $-0.5 \pm 8.0$ & $-16.2 \sim 15.2$ & $2.4 \pm 11.4$ & $-19.9 \sim 24.7$ \\
\hline
\end{tabular}

Abbreviations: DBP, diastolic blood pressure; MBP, mean blood pressure; NTG, sublingual nitroglycerin; PP, pulse pressure; PVP, pulse volume plethysmography; SBP, systolic blood pressure; SBP2, pressure value of the late systolic shoulder produced by wave reflections.

All values are shown as mean \pm s.d.

${ }^{*} P<0.05 ;{ }^{* *} P<0.01 ; * * * P<0.001$

${ }^{a}$ Calibrated by cuff SBP and DBP.

Table 3 Bias and confidence limits of estimation for aortic sbp by brachial and cuff SBP and SBP2 and the prediction models in the validation group $(n=50)$

\begin{tabular}{|c|c|c|c|}
\hline & Bias, $\mathrm{mm} \mathrm{Hg}$ & $\begin{array}{c}95 \% \mathrm{Cl} \\
\mathrm{mm} \mathrm{Hg}\end{array}$ & $r$ \\
\hline \multicolumn{4}{|l|}{ Brachial SBP } \\
\hline Baseline & 7.8 & $-2.6 \sim 18.2$ & $0.97 * * *$ \\
\hline After NTG & 11.1 & $-2.4 \sim 24.6$ & $0.92 * * *$ \\
\hline \multicolumn{4}{|c|}{ Brachial SBP2 } \\
\hline Baseline & 2.5 & $-6.1 \sim 11.1$ & $0.98 * * *$ \\
\hline After NTG & 3.5 & $-8.1 \sim 15.1$ & $0.95^{* * *}$ \\
\hline \multicolumn{4}{|c|}{ Invasive Multivariate Model } \\
\hline Baseline & -0.3 & $-7.9 \sim 7.3$ & $0.98 * * *$ \\
\hline After NTG & 1.3 & $-8.3 \sim 10.9$ & $0.96 * * *$ \\
\hline \multicolumn{4}{|l|}{ Cuff SBP } \\
\hline Baseline & 3.3 & $-13.0 \sim 19.6$ & $0.92^{* * *}$ \\
\hline After NTG & 6.7 & $-13.3 \sim 26.7$ & $0.81 * * *$ \\
\hline \multicolumn{4}{|l|}{ PVP SBP2a } \\
\hline Baseline & -0.5 & $-16.2 \sim 15.2$ & $0.93 * * *$ \\
\hline After NTG & 2.4 & $-17.4 \sim 22.2$ & $0.81^{* * *}$ \\
\hline \multicolumn{4}{|c|}{ Noninvasive Multi-variate Model } \\
\hline Baseline & -0.3 & $-15.0 \sim 14.4$ & $0.94 * * *$ \\
\hline After NTG & 0.2 & $-15.1 \sim 15.5$ & $0.90 * * *$ \\
\hline
\end{tabular}

Abbreviations: Bias, difference between the pressure value and aortic SBP; NTG, sublingual nitroglycerin; PVP, pulse volume plethysmography; r, correlation coefficient; SBP, systolic blood pressure; SBP2, pressure value of the late systolic shoulder produced by wave reflections. $* * * P<0.001$.

aCalibrated by cuff SBP and DBP.

Compared with the invasive brachial SBP, the cuff SBP showed a smaller bias but greater scattering in the estimation of invasive aortic SBP (Table 3, Figure 3a). Although the bias substantially reduced with
PVP SBP2, the agreement limits remained large (Table 3, Figure 3b). With the noninvasive model, the agreement limits further reduced (Table 3). The overall agreement limits for steady state and after NTG were -15.0 to $14.8 \mathrm{~mm} \mathrm{Hg}$ (Figure 3c). The mean difference between the predicted and observed aortic SBP was not significantly greater than $0 \mathrm{~mm} \mathrm{Hg}$, and was significantly smaller than that between PVP SBP2 and aortic SBP at baseline and after NTG (all $P<0.01$ ) (Table 3, Figure $3 \mathrm{c}$ ). The correlation between the measured and predicted aortic SBP by the noninvasive prediction model (Figure 3c) was significantly better than that by PVP SBP2 (Figure $3 \mathrm{~b})(P<0.001)$. Similar to the invasive model, the Bland-Altman analysis indicated that there was no systemic bias of estimation by the noninvasive model with different levels of estimated values.

\section{Technical issues related to the PVP waveform analysis}

Inflection point identification. In this study population of 100 subjects with a total of 200 recorded PVP waveforms (baseline and after NTG administration), an inflection point (for the determination of SBP2) on the PVP waves was visually discernible in 89 subjects at baseline, and 51 subjects after NTG (70\% of all PVP waveforms), respectively. Although SBP2 was not always visually discernible on the PVP waves, all SBP2 could be objectively identified by determining the zero-crossing timings of the fourth derivative of the PVP wave (Supplementary Figure S1). The time interval $(231.4 \pm 33.4 \mathrm{~ms}$ ) from foot to peak of reflection wave (SBP2) on PVP waveforms was slightly longer than the corresponding time interval $(216.5 \pm 40.0 \mathrm{~ms})$ on the simultaneously obtained aortic pressure waves. The estimated potential error in the prediction of aortic SBP from the identification of SBP2 on PVP waves alone was $-0.7 \pm 1.7 \mathrm{~mm} \mathrm{Hg}$.

End-systole identification. In the same way, the time interval $(348.2 \pm 38.3 \mathrm{~ms})$ from foot to end-systole on PVP waves was longer 

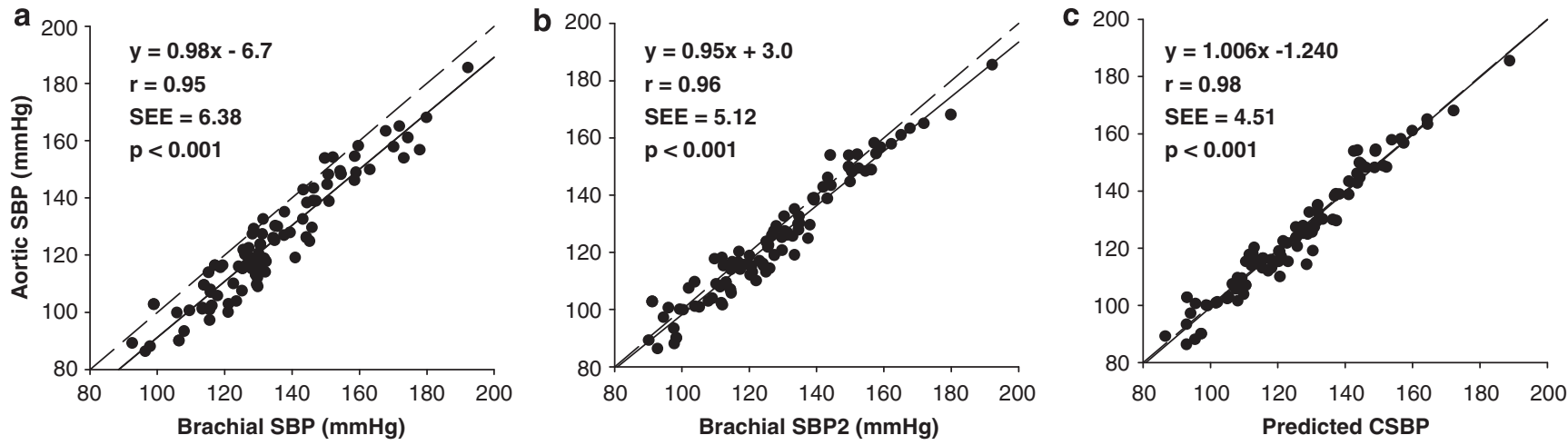

by Invasive Model (mmHg)
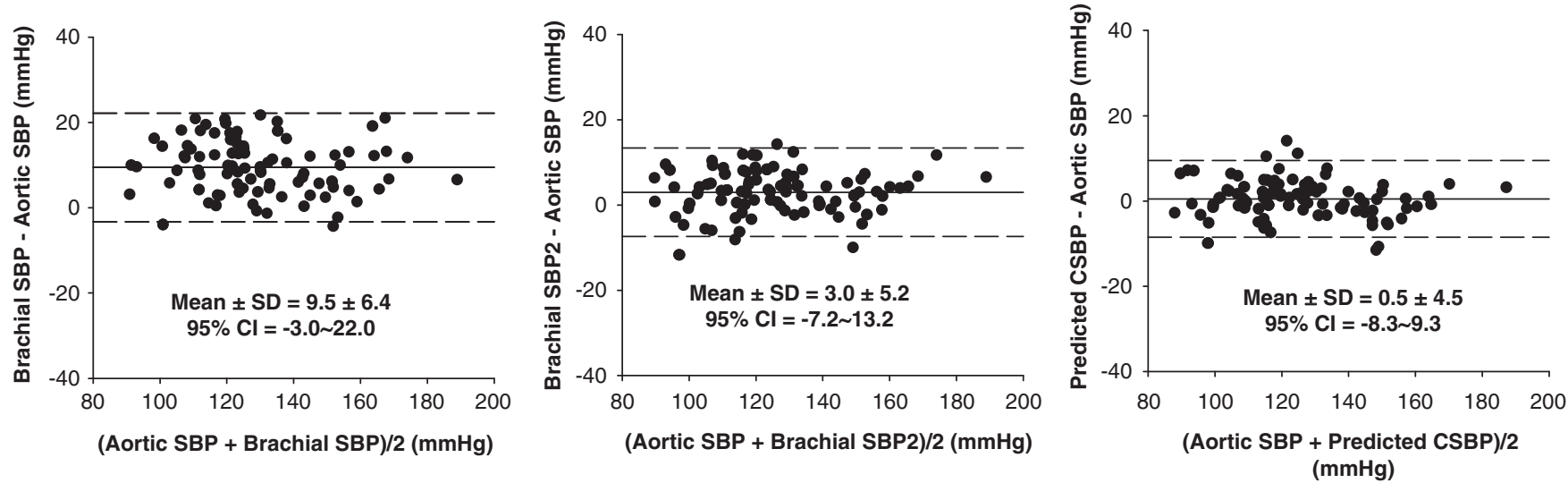

Figure 2 Bland-Altman analyses in the Validation Group ( $n=50)$. Data combine baseline and after sublingual nitroglycerin administration measurements. (a) agreement between invasive brachial systolic blood pressure (SBP) and measured aortic SBP; (b) agreement between invasive brachial pressure value of the late systolic shoulder (SBP2) and measured aortic SBP; (c) agreement between measured and predicted aortic SBP by the invasive brachial pressure wave prediction model; Dashed lines indicate the boundaries of two s.d. of the differences. Solid line indicates mean of the differences. $\mathrm{Cl}$, confidence interval.

than the corresponding time interval $(314.9 \pm 36.2 \mathrm{~ms})$ on the simultaneously obtained aortic pressure waves. The estimated potential error in the prediction of aortic SBP from the identification of endsystolic pressure on PVP waves alone was $0.3 \pm 1.0 \mathrm{~mm} \mathrm{Hg}$. When applying the aortic timings of both SBP2 and end-systolic pressure on the PVP waves, the estimated potential error in the prediction of aortic SBP was $-0.4 \pm 2.0 \mathrm{~mm} \mathrm{Hg}$.

\section{DISCUSSION}

This study successfully developed and validated a novel method to estimate the central aortic SBP by analyzing the oscillometric signals from an oscillometric blood pressure monitor. The study confirmed that SBP2 measured from a peripheral pressure wave approximates central aortic SBP, ${ }^{11-14}$ and extended that approximation of brachial SBP2 to aortic SBP can further be improved by the comprehensive analysis of the radial or brachial pressure waves. The study also clearly showed that the major source of errors in the noninvasive estimation of central blood pressure was from the variability of the oscillometric cuff blood pressure measurement, which was particularly prominent after the administration of vasoactive agents.

Brachial SBP correlated with aortic SBP significantly $(r=0.95$, $P<0.001$ in the Validation Group, Figure 2a) but the estimation of aortic SBP with brachial SBP alone generated large individual variation (Figure 2a). Combining brachial SBP2 with other parameters from the brachial waveform analysis further improved the prediction $(r=0.98, P<0.001$, Figure 2c). Although the incremental contribution from the waveform analysis other than the identification of SBP2 may appear small $(4.2 \%)$, it was statistically significant $(P<0.001)$ and may be critical for the noninvasive application. The seemingly small prediction improvement may help reduce the large individual variation introduced from the inaccuracy of oscillometric SBP and DBP to a clinically acceptable level.

The multivariate invasive and noninvasive brachial pressure wave prediction models clearly showed that brachial SBP2 was the predominant correlate of aortic SBP, with additional independent contribution from correlates of the total arterial compliance. ${ }^{17}$ Therefore, our results support that peripheral SBP2 closely approximates central aortic SBP, ${ }^{11-14}$ and that central SBP may be increased by increased augmentation index, decreased arterial compliance, and increased arterial stiffness. ${ }^{3,19}$

In this study, heart rate was not significantly associated with central aortic SBP and did not contribute to the prediction models. Although increased heart rate is associated with decreased augmentation index ${ }^{20}$ decreased arterial compliance, ${ }^{21}$ and increased arterial stiffness, ${ }^{22}$ incremental pacing was not associated with change of the central SBP. ${ }^{20}$ This is probably because heart-rate-associated changes in wave reflections and arterial compliance have opposite effect on the central SBP. Because the variation of heart rate was not large enough in this study, assessment of the impact of heart rate on central SBP may require further studies.

The performance of the current PVP method is considered clinically acceptable because the limits of agreement $(-15.0$ to $14.8 \mathrm{~mm} \mathrm{Hg})$ 

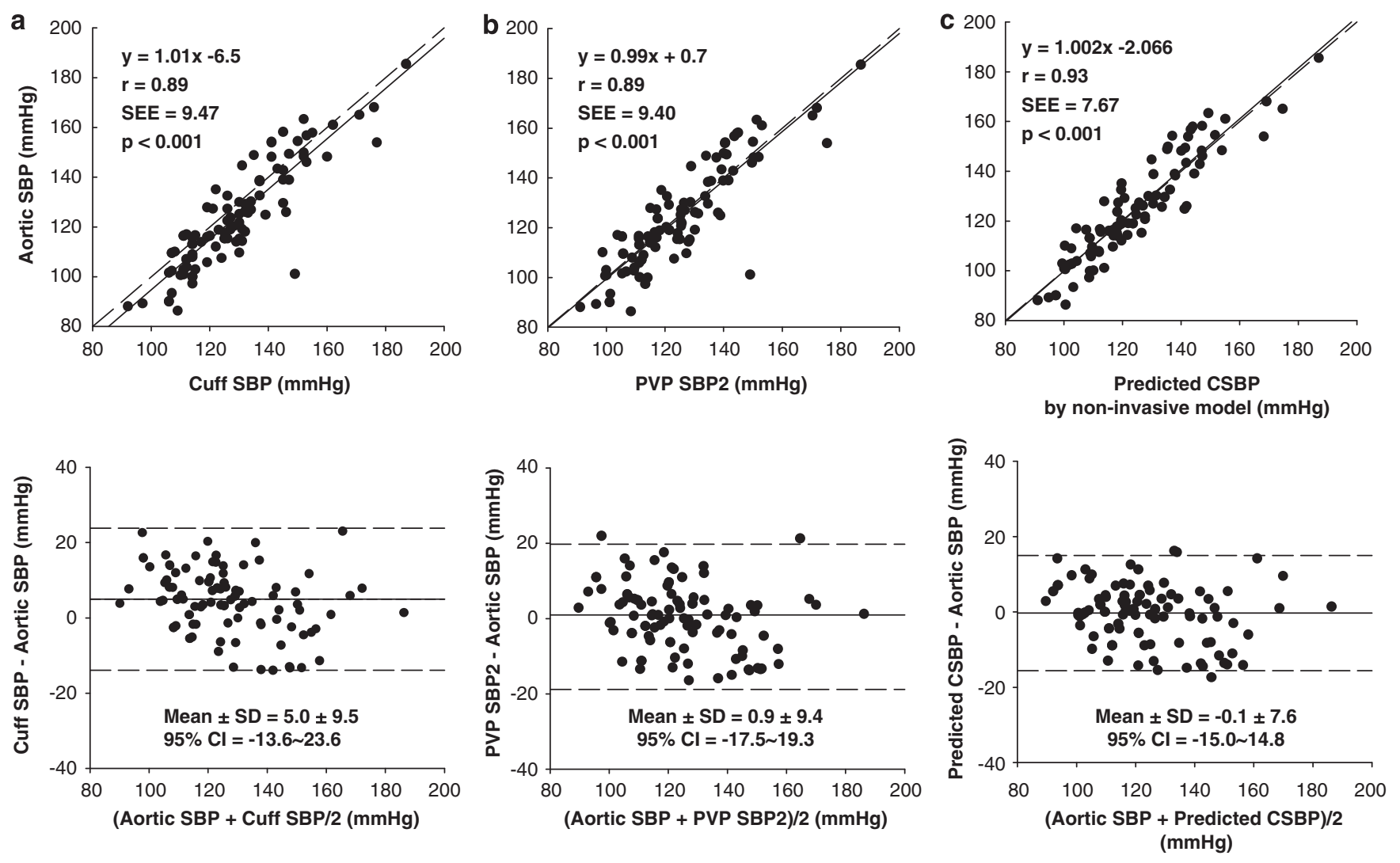

Figure 3 Bland-Altman analyses in the Validation Group ( $n=50)$. Data combine baseline and after sublingual nitroglycerin administration measurements. (a) agreement between cuff systolic blood pressure (SBP) and measured aortic SBP; (b) agreement between the late systolic shoulder of the calibrated pulse volume plethysmography waveform (PVP SBP2) and measured aortic SBP; (c) agreement between measured and predicted aortic SBP by the non-invasive brachial pressure wave prediction model; Dashed lines indicate the boundaries of two s.d. of the differences. Solid line indicates mean of the differences. $\mathrm{Cl}$, confidence interval.

were not larger than those for the cuff SBP vs. invasive brachial SBP $(-21.2$ to $12.2 \mathrm{~mm} \mathrm{Hg}$ ) and the mean difference (Figure $3 \mathrm{c}$ ) between the predicted and measured aortic SBP was $\pm 5 \mathrm{~mm} \mathrm{Hg}$ or less with a s.d. of $8 \mathrm{~mm} \mathrm{Hg}$ or less (Association for the Advancement of Medical Instrumentation criteria). ${ }^{23}$ The mean difference and s.d. were also comparable with those of the radial tonometry using a generalized transfer function (mean difference $=-13.3 \pm 15.1 \mathrm{~mm} \mathrm{Hg}$ ) ${ }^{24}$ or by identifying the radial SBP2 (mean difference $=-11.7 \pm 7.1 \mathrm{~mm} \mathrm{Hg}$ ). ${ }^{14}$ The large s.d. between the predicted and observed aortic SBP in the present and previous studies were mainly introduced by the large variations between the indirect cuff blood pressure and the direct invasive brachial blood pressure. ${ }^{14,24}$ Large variations between the indirect and direct blood pressure measurements have been well recognized. ${ }^{25-28}$ A previous study assessing the accuracy of indirect blood pressure measurements at steady state clearly showed that the indirect methods underestimated SBP and overestimated DBP compared with the direct method, with s.d. of $10.5 / 7.6 \mathrm{~mm} \mathrm{Hg}$ (SBP/DBP), 9.7/8.9 $\mathrm{mm} \mathrm{Hg}$ and 8.1/6.5 $\mathrm{mm} \mathrm{Hg}$ with the oscillometric method, microphone method and the auscultatory method, respectively. ${ }^{26}$ In this study, the accuracy of the oscillometric blood pressure monitor was reasonably good as shown by s.d. for SBP/DBP of 7.2/ $6.1 \mathrm{~mm} \mathrm{Hg}$ at steady state and 9.7/8.1 $\mathrm{mm} \mathrm{Hg}$ after NTG administration, respectively (Table 2 ).

Although brachial blood pressure measurements using oscillometric or Korotkov method cuff sphygmomanometers are limited in accuracy and precision, ${ }^{25}$ the oscillometric arm blood pressure monitors are valuable and indispensable for the management of hypertension and are recommended for home blood pressure monitoring. ${ }^{29}$ Our study reconfirmed that the oscillometric method substantially underestimates brachial SBP and overestimates brachial DBP, while providing reasonably good estimates of the brachial MBP. ${ }^{14,26,30}$ Although all current noninvasive methods for the estimation of aortic pressure are affected by the limited accuracy of the oscillometric blood pressure monitors, ${ }^{31,32}$ the clinical values of the central blood pressure estimates remain evident. $5,7,8$

Because brachial SBP2 represents a very significant weight in both the invasive and noninvasive prediction models, the accurate identification of SBP2 on a peripheral pulse without visible late systolic peak may deserve concerns. The identification of SBP2 on a radial pressure waveform may be difficult when it is close to the end-systolic pressure as with nitroglycerin, in youth, with tachycardia, and in systolic heart failure. ${ }^{33}$ In a large cohort study of 10269 participants, SBP2 on a pressure waveform obtained by a high-fidelity tonometric contact sensor on the radial artery could be identified by the inbuilt software in $98.2 \%$ of the recordings with a strong correlation $(r=0.99$, $P<0.001)$ with the noninvasively derived central SBP. ${ }^{34}$ Estimation of central SBP from radial SBP2 was less accurate at low SBP values and the estimation could be further improved by inclusion parameters such as augmentation index, heart rate, MBP, aortic pulse wave velocity and underline medical history. ${ }^{34}$ Thus, their and our results clearly show that the identification of peripheral SBP2 is feasible in most subjects and the approximation of central SBP by peripheral 
SBP2 can be further improved with various strategies, such as the comprehensive waveform analysis proposed by this study.

\section{Limitations of this study}

Although the Generation and Validation Groups were independently enrolled, their characteristics were similar. Therefore, the external validity of the proposed invasive and noninvasive prediction models might still be limited. The recordings of the invasive brachial and aortic pressure waves were not simultaneous. Because there were only small differences between the measured brachial and aortic MBP and DBP and the performance of the invasive model was comparable with the generalized transfer function approach for the simultaneously recorded aortic and radial pressure waveforms, ${ }^{35}$ the hemodynamic changes between the measurements at the two arterial sites might be negligible. Although the PVP waveform has limited frequency response and its inflection point after NTG was subjectively visible in only half of the measurements in this study, it could be automatically detected in all cases when using the well-documented zerocrossing timings of the fourth derivative of the pressure wave. ${ }^{2}$ The robustness of the current fourth derivative method has been partially validated in this study by showing a small error in the measurement of the interval from wave foot to peak of the reflection wave on the PVP wave with reference to the equivalent interval on the simultaneously recorded aortic pressure, and the small impact on the prediction of central SBP from the error. The observed accuracy of the estimation for central SBP by the noninvasive prediction model in the Validation Group also implies that the accuracy of the fourth derivative method is acceptable. Because the proposed models were generated from and validated in subjects receiving diagnostic catheterization in supine position, generalization of the models to younger subjects and those in sitting position may require further validation.

In conclusion, central aortic systolic pressure could be estimated with acceptable accuracy and precision by analysis of the noninvasive brachial pressure wave from an oscillometric blood pressure monitor. This innovation can be built into most oscillometric blood pressure monitors and may expedite the use of central blood pressure in the management of hypertension.

\section{CONFLICT OF INTEREST}

Omron Healthcare has pay royalties to National Yang-Ming University to own priority to negotiate technology licenses or transfers. Otherwise, no conflict of interest declared.

\section{ACKNOWLEDGEMENTS}

We thank the Omron Healthcare, Kyoto, Japan, for free loan of the VP-2000 apparatus. This work was supported by National Science Council (NSC 962314-B-010 -035 -MY3), Ministry of Education, Aim for the Top University Plan (96A-D-D131), and an intramural grant from the Taipei Veterans General Hospital (Grant No. V99C1-091).

1 Krooker EJ, Wood EH. Comparison of simultaneously recorded central and peripheral arterial pressure pulses during rest, exercise and tilted position in man. Circ Res 1955; 3: 623-632.

2 Takazawa K, Tanaka N, Takeda K, Kurosu F, Ibukiyama C. Underestimation of vasodilator effects of nitroglycerin by upper limb blood pressure. Hypertension 1995; 26: 520-523.

3 Segers P, Mahieu D, Kips J, Rietzschel E, De Buyzere M, De Bacquer D, Bekaert S, De Backer G, Gillebert T, Verdonck P, Van Bortel L. Amplification of the pressure pulse in the upper limb in healthy, middle-aged men and women. Hypertension 2009; 54: 414-420.

4 Kelly RP, Gibbs HH, O'Rourke MF, Daley JE, Mang K, Morgan JJ, Avolio AP. Nitroglycerin has more favourable effects on left ventricular afterload than apparent from measurement of pressure in a peripheral artery. Eur Heart J 1990; 11: 138-144.
5 Roman MJ, Devereux RB, Kizer JR, Lee ET, Galloway JM, Ali T, Umans JG, Howard BV. Central pressure more strongly relates to vascular disease and outcome than does brachial pressure: the Strong Heart Study. Hypertension 2007; 50: 197-203.

6 Wang KL, Cheng HM, Chuang SY, Spurgeon HA, Ting CT, Lakatta EG, Yin FCP, Chou P, Chen $\mathrm{CH}$. Central or peripheral systolic or pulse pressure: which best relates to target organs and future mortality? J Hypertens 2009; 27: 461-467.

7 Jankowski P, Kawecka-Jaszcz K, Czarnecka D, Brzozowska-Kiszka M, Styczkiewicz K, Loster M, Kloch-Badelek M, Wilinnski J, Curylo AM, Dudek D. Pulsatile but not steady component of blood pressure predicts cardiovascular events in coronary patients. Hypertension 2008; 51: 848-855.

8 Williams B, Lacy PS, Thom SM, Cruickshank K, Stanton A, Collier D, Hughes AD, Thurston H, O'Rourke M. Differential impact of blood pressure-lowering drugs on central aortic pressure and clinical outcomes: principal results of the Conduit Artery Function Evaluation (CAFE) study. Circulation 2006; 113: 1213-1225.

9 Chen $\mathrm{CH}$, Nevo E, Fetics B, Pak PH, Yin FCP, Maughan WL, Kass DA. Estimation of central aortic pressure waveform by mathematical transformation of radial tonometry pressure: validation of generalized transfer function. Circulation 1997; 95: 1827-1836.

10 Karamanoglu M, O'Rourke MF, Avolio AP, Kelly RP. An analysis of the relationship between central aortic and peripheral upper limb pressure waves in man. Eur Heart $J$ 1993; 14: 160-167.

11 Pauca AL, Kon ND, O'Rourke MF. The second peak of the radial artery pressure wave represents aortic systolic pressure in hypertensive and elderly patients. $\mathrm{Br} \mathrm{J}$ Anaesth 2004; 92: 651-657.

12 Adji A, O'Rourke MF. Determination of central aortic systolic and pulse pressure from the radial artery pressure waveform. Blood Press Monit 2004; 9: 115-121.

13 Munir S, Guilcher A, Kamalesh T, Clapp B, Redwood S, Marber M, Chowienczyk P. Peripheral augmentation index defines the relationship between central and peripheral pulse pressure. Hypertension 2008; 51: 112-118.

14 Takazawa K, Kobayashi H, Shindo N, Tanaka N, Yamashina A. Relationship between radial and central arterial pulse wave and evaluation of central aortic pressure using the radial arterial pulse wave. Hypertens Res 2007; 30: 219-228.

15 Yamashina A, Tomiyama H, Takeda K, Tsuda H, Arai T, Hirose K, Koji Y, Hori S, Yamamoto Y. Validity, reproducibility, and clinical significance of noninvasive brachialankle pulse wave velocity measurement. Hypertens Res 2002; 25: 359-364.

16 Darling RC, Raines JK, Brener BJ, Austen WG. Quantitative segmental pulse volume recorder: a clinical tool. Surgery 1972; 72: 873-877.

17 Liu Z, Brin KP, Yin FCP. Estimation of total arterial compliance: an improved method and evaluation of current methods. Am J Physiol Heart Circ Physiol 1986; 251: $\mathrm{H} 588-\mathrm{H} 600$

18 Kleinbaum DG, Kupper LL, Muller KE, Nizam A. Applied Regression Analysis and Multivariable Methods, 3rd ed. Duxburry Press: New York, 1997.

19 Protogerou AD, Papaioannou TG, Lekakis JP, Blacher J, Safar ME. The effect of antihypertensive drugs on central blood pressure beyond peripheral blood pressure. Part I: (patho)-physiology, rationale and perspective on pulse pressure amplification. Curr Pharm Des 2009; 15: 267-271.

20 Wilkinson IB, MacCallum H, Flint L, Cockcroft JR, Newby DE, Webb DJ. The influence of heart rate on augmentation index and central arterial pressure in humans. $J$ Physiol 2000; 525(Part 1): 263-270.

21 Liang YL, Gatzka CD, Du XJ, Cameron JD, Kingwell BA, Dart AM. Effects of heart rate on arterial compliance in men. Clin Exp Pharmacol Physiol 1999; 26: 342-346.

22 Papaioannou TG, Vlachopoulos CV, Alexopoulos NA, Dima I, Pietri PG, Protogerou AD, Vyssoulis GG, Stefanadis $\mathrm{CI}$. The effect of heart rate on wave reflections may be determined by the level of aortic stiffness: clinical and technical implications. Am J Hypertens 2008; 21: 334-340.

23 White WB, Berson AS, Robbins C, Jamieson MJ, Prisant LM, Roccella E, Sheps SG. National standard for measurement of resting and ambulatory blood pressures with automated sphygmomanometers. Hypertension 1993; 21: 504-509.

24 Cloud GC, Rajkumar C, Kooner J, Cooke J, Bulpitt CJ. Estimation of central aortic pressure by SphygmoCor requires intra-arterial peripheral pressures. Clin Sci (London) 2003; 105: 219-225.

25 van Montfrans GA. Oscillometric blood pressure measurement: progress and problems. Blood Press Monit 2001; 6: 287-290.

26 Ochiai H, Miyazaki N, Miyata T, Mitake A, Tochikubo O, Ishii M. Assessment of the accuracy of indirect blood pressure measurements. Jpn Heart J 1997; 38: 393-407.

27 Manios E, Vemmos K, Tsivgoulis G, Barlas G, Koroboki E, Spengos K, Zakopoulos N. Comparison of noninvasive oscillometric and intra-arterial blood pressure measurements in hyperacute stroke. Blood Press Monit 2007; 12: 149-156.

28 Araghi A, Bander JJ, Guzman JA. Arterial blood pressure monitoring in overweight critically ill patients: invasive or noninvasive? Crit Care 2006; 10: R64.

29 Pickering TG, Miller NH, Ogedegbe G, Krakoff LR, Artinian NT, Goff D. Call to action on use and reimbursement for home blood pressure monitoring: executive summary. a joint scientific statement from the American Heart Association, American Society of Hypertension, and Preventive Cardiovascular Nurses Association. Hypertension 2008; 52: 1-9.

30 Smulyan H, Sheehe PR, Safar ME. A preliminary evaluation of the mean arterial pressure as measured by cuff oscillometry. Am J Hypertens 2008; 21: $166-171$. 
31 Smulyan H, Siddiqui DS, Carlson RJ, London GM, Safar ME. Clinical utility of aortic pulses and pressures calculated from applanated radial-artery pulses. Hypertension 2003; 42: 150-155.

32 Protogerou AD, Papaioannou TG, Blacher J, Papamichael CM, Lekakis JP, Safar ME. Central blood pressures: do we need them in the management of cardiovascular disease? Is it a feasible therapeutic target? J Hypertens 2007; 25: 265-272.

33 O'Rourke MF, Adji A. Basis for use of central blood pressure measurement in office clinical practice. J Am Soc Hypertens 2008; 2: 28-38.
34 Hickson SS, Butlin M, Mir FA, Graggaber J, Cheriyan J, Khan F, Grace AA, Yasmin, Cockcroft JR, Wilkinson IB, McEniery CM. The accuracy of central SBP determined from the second systolic peak of the peripheral pressure waveform. J Hypertens 2009; 27: 1784-1788.

35 Pauca AL, O'Rourke MF, Kon ND. Prospective evaluation of a method for estimating ascending aortic pressure from the radial artery pressure waveform. Hypertension 2001; 38: 932-937.

Supplementary Information accompanies the paper on Hypertension Research website (http://www.nature.com/hr) 